\title{
Universal portfolios generated by rational functions
}

\author{
Kee Seng Kuang ${ }^{1 *}$, Choon Peng Tan ${ }^{1}$, and Yann Ling Goh ${ }^{1}$ \\ ${ }^{1}$ Department of Mathematical and Actuarial Sciences, Universiti Tunku Abdul Rahman, Jalan Sungai \\ Long, 43000, Kajang, Selangor, Malaysia
}

\begin{abstract}
The $f$-divergence of Csiszar is defined for a non-negative convex function on the positive axis. A pseudo $f$-divergence can be defined for a convex function not satisfying the usual requirements. A rational function where both the numerator and the denominator are non-integer polynomials will be used to generate universal portfolios. Five stock-price data sets from the local stock exchange are selected for the empirical study. Empirical results are obtained by running the generated portfolios on these data sets. The empirical results demonstrate that it is possible for the investors to increase their wealth by using the portfolios in investment.
\end{abstract}

\section{Introduction}

Cover and Ordentlich [1] introduced a general method to generate universal portfolio by weighting the current and past relatives in a stock portfolio utilizing the moments of a probability distribution with emphasis on the Dirichlet distribution. However, the CoverOrdentlich universal portfolio requires a substantial amount of computer memory and implementation time. To surpass this difficult, Tan [2] proposed the finite-order universal portfolio to save computer memory and implementation time. Empirical studies show that the performance of finite-order portfolios can match the performance of the CoverOrdentlich universal portfolios. Helmbold et al. [3] proposed a method to generate a universal portfolio using the zero-gradient set of an objective function consist of KullbackLeibler divergence (one of the $f$-divergence) of two portfolio vectors. Tan and Kuang [4] studied method to generate universal portfolios by $f$-divergence and Bregman divergences.

The $f$-divergence is generated by a non-negative convex function $f(t)$ on the positive axis. A pseudo $f$-divergence is defined for a convex function which does not satisfying the usual requirements. Tan and Kuang [5] proposed the use of $f$-divergence which is defined for two types of convex functions to generate universal portfolios. The first type is a nonnegative function convex on a subset of the positive axis. The second one is a function convex on the positive axis but non-negative on a subject of axis. The universal portfolio generated by $f$-divergence using second type of convex functions defined above will be derived. Then the empirical result will be compared with the well-known Helmbold's Universal Portfolio.

*Corresponding author: kuangks@utar.edu.my 


\section{Some basic theory}

\subsection{Definition}

Investment in a stock market with $m$ number of stocks is considered. The market is described by a sequence of price relative vector $\boldsymbol{x}_{n}=\left(x_{n i}\right)$ on the $n^{\text {th }}$ trading day. The $x_{n i}$ is the price relative of the $i^{\text {th }}$ stock which is defined as the ratio of the closing price of stock to its opening price on day $n$, for $i=1,2, \cdots, m$. A portfolio $\boldsymbol{b}_{n}=\left(b_{n i}\right)$ is the portfolio strategy used on the $n^{\text {th }}$ trading day where $b_{n i}$ is the weightage of the wealth at the beginning of the $n^{\text {th }}$ trading day invested on stock $i$, for $i=1,2, \cdots, m, 0 \leq b_{n i} \leq 1$ and $\sum_{i=1}^{m} b_{n i}=1$. The initial wealth invested is assumed to be 1 unit. The wealth $S_{n}\left(\boldsymbol{x}_{n}\right)$ at the end of the $n^{\text {th }}$ trading day is

$$
S_{n}\left(x_{n}\right)=\prod_{j=1}^{n} \boldsymbol{b}_{j}^{t} \boldsymbol{x}_{j}=\prod_{j=1}^{n}\left(\sum_{i=1}^{m} b_{j i} x_{j i}\right) .
$$

Let $f(t)$ define a convex function on $(0, \infty)$, is strictly convex at $t=1$, and $f(1)=0$. The Csiszar's $f$-divergence of two probability distributions $\boldsymbol{p}=\left(p_{i}\right)$ and $\boldsymbol{q}=\left(q_{i}\right)$ is defined as

$$
D_{f}(\boldsymbol{p} \| \boldsymbol{q})=\sum_{i=1}^{m} q_{i} f\left[\frac{p_{i}}{q_{i}}\right] .
$$

The $f$-divergence given in Equation (2) is also known as the $f$-disparity difference between two probability distribution $\boldsymbol{p}=\left(p_{i}\right)$ and $\boldsymbol{q}=\left(q_{i}\right)$ given the $f(t)$ is a $f$-disparity function which fulfill the followings. A continuous function of $f(t)$ on $(0, \infty)$ is a $f$ disparity function if

i. $f(t)$ is a decreasing function for $0<t<1$.

ii. $\quad f(t)$ is an increasing function for $t>1$.

iii. $f(1)=0$.

iv. $\quad f(0)$ is determined by the continuous extension of $f(t)$ (see [6], pg. 29)

In the statistical inference literature, the $f$-disparity function is also known as the phidisparity function. The convex function $f(t)$ from the $f$-divergence is an the $f$-disparity function.

For two portfolio vectors $\boldsymbol{b}_{n+1}$ and $\boldsymbol{b}_{n}$, the $f$-divergence is defined as

$$
D_{f}\left(\boldsymbol{b}_{n+1} \| \boldsymbol{b}_{n}\right)=\sum_{i=1}^{m} b_{n i} f\left[\frac{b_{n+1, i}}{b_{n i}}\right] .
$$

\section{Main results}

Let $f(t)$ define a given $f$-disparity difference. Then the Type-1 universal portfolio $\boldsymbol{b}_{n+1}$ generated by $f(t)$ corresponding to the object function 
is given by

$$
\begin{gathered}
\widehat{F}\left(\boldsymbol{b}_{n+1} ; \lambda\right)=\eta\left[\log \left(\boldsymbol{b}_{n}^{t} \boldsymbol{x}_{n}\right)+\left(\frac{\boldsymbol{b}_{n+1}^{t} \boldsymbol{x}_{n}}{\boldsymbol{b}_{n}^{t} \boldsymbol{x}_{n}}\right)-1\right]-D_{f}\left(\boldsymbol{b}_{n+1} \| \boldsymbol{b}_{n}\right) \\
+\lambda\left(\sum_{j=1}^{m} b_{n+1, j}-1\right)=0
\end{gathered}
$$

where $\lambda$ is the Lagrange multiplier and the parameter $\eta$ and $\xi$ are assumed to be constants.

A non-negative, continuously differentiable convex function $g(t)$ on $(0, \infty)$ may not satisfy the condition $g(1)=0$. The function $g(t)$ is then translated by $g(1)$ to satisfy $f(1)=0$, e.g. $f(t)=g(t)-g(1)$. Consider function $f(t)=g(t)-g(1)$ on $(0, \infty)$ where function $g(t)$ is a given continuously differentiable, non-negative convex function on $(0, \infty)$. For a translated convex function $g(t)$, a pseudo $f$-divergence can be defined by

$$
f(t)=g(t)-g(1)
$$

by substituting $f(t)$ in Equation (2) by Equation (6).

For $t \geq 0$, consider the convex function

$$
g(t)=\frac{d_{1}}{(\beta+1)}\left(t+c_{1}\right)^{\beta+1}+\frac{d_{2}}{(1-\beta)} \frac{1}{\left(t+c_{2}\right)^{\beta-1}},
$$

For constants $c_{1}>0, c_{2}>0, d_{1}>0, d_{2}<0$ and the parameter $\beta>1$.

For $\beta=1, g(t)$ is defined as

$$
g(t)=\frac{d_{1}}{2}\left(t+c_{1}\right)^{2}+d_{2} \log \left(t+c_{2}\right)
$$

A pseudo $f$-divergence is defined by Equation (2) for $f(t)$ and $g(t)$ given by Equation (6) and Equation (7) or Equation (8) respectively.

\subsection{Proposition}

Let the convex function $g(t)$ given by Equation (7) or Equation (8), $f(t)$ given by Equation (6) and the objective function Equation (4).

\subsubsection{Proposition 1}

For $c_{1}=c_{2}=c>0$ and $\beta>1$, a valid version of the universal portfolio $\boldsymbol{b}_{n+1}$ generated by $f(t)$ is given by

for $j=1,2, \cdots, m$ where

$$
b_{n+1, i}=\frac{b_{n i}\left\{\left[\frac{1}{2 d_{1}}\left(v_{i}+\sqrt{v_{i}^{2}-4 d_{1} d_{2}}\right)\right]^{\frac{1}{\beta}}-c\right\}}{\sum_{j=1}^{m} b_{n j}\left\{\left[\frac{1}{2 d_{1}}\left(v_{j}+\sqrt{v_{j}^{2}-4 d_{1} d_{2}}\right)\right]^{\frac{1}{\beta}}-c\right\}}
$$

and $\left[\frac{1}{2 d_{1}}\left(v_{i}+\sqrt{v_{i}^{2}-4 d_{1} d_{2}}\right)\right]^{\frac{1}{\beta}}>c$ for selected values of $v_{i}$ and $c$. 


\subsubsection{Proposition 2}

For $\beta=1$, a valid version of the universal portfolio $\boldsymbol{b}_{n+1}$ generated by $f(t)$ is given by $b_{n+1, i}$

$$
\left.=\frac{b_{n i}\left\{+\frac{1}{2 d_{1}} \sqrt{\left\{v_{i}-d_{1}\left(c_{1}+c_{2}\right)\right\}^{2}-4 d_{1}\left[d_{1} c_{1} c_{2}+d_{2}-v_{i} c_{2}\right]}\right\}}{\frac{1}{2 d_{1}}\left[v_{j}-d_{1}\left(c_{1}+c_{2}\right)\right]}\right\}
$$

for $i=1,2, \cdots, m$, where $v_{i}$ is given by Equation (10) and the numerator of Equation (11) is positive for selected values of $v_{i}, c_{1}$ and $c_{2}$.

\subsection{Proof}

The universal portfolio $\boldsymbol{b}_{n+1}$ generated by the pseudo $f$-divergence Equation (2) is given by

$$
f^{\prime}(t)=g^{\prime}(t)=d_{1}(t+c)^{\beta}+d_{2}(t+c)^{-\beta}=v_{i}
$$

for $i=1,2, \cdots, m$ from Equation (6), Equation (7), Equation (5) and Equation (10) where $t=\frac{b_{n+1, i}}{\text { bni }}$.

Simplifying the Equation (12),

$$
d_{1}(t+c)^{2 \beta}-v_{i}(t+c)^{\beta}+d_{2}=0
$$

Solving the quadratic in $(t+c)$ and taking the positive root,

$$
t=\left[\frac{1}{2 d_{1}}\left(v_{i}+\sqrt{v_{i}^{2}-4 d_{1} d_{2}}\right)\right]^{\frac{1}{\beta}}-c .
$$

Then, normalizing Equation (14) leads to Equation (9).

Next, the universal portfolio $\boldsymbol{b}_{n+1}$ generated by the pseudo $f$-divergence Equation (2) is given by

$$
f^{\prime}(t)=g^{\prime}(t)=d_{1}(t+c)+d_{2}\left(t+c_{2}\right)^{-1}=v_{i}
$$

for $i=1,2, \cdots, m$ from Equation (6), Equation (8), Equation (5) and Equation (10) where $t=\frac{b_{n+1, i}}{\text { bni }}$.

Simplifying the Equation (15),

$$
d_{1}\left(t+c_{1}\right)\left(t+c_{2}\right)+d_{2}-v_{i}\left(t+c_{2}\right)=0
$$

for $i=1,2, \cdots, m$.

Solving the quadratic in $t$ and normalizing $b_{n+1, i}$, the universal portfolio (11) is obtained.

\section{Empirical results}

Table 1 shows the list of Malaysian Companies chosen from Kuala Lumpur Stock Exchange (KLSE) for empirical study. These data are collected from Bloomberg for the period $3^{\text {rd }}$ January 2005 to $4^{\text {th }}$ September 2015 . Each stock consists of 2500 trading days. Then stocks are grouped into stock-price data sets, J, K, L, M and N. 
Table 3 and 4 show the result obtained after the universal portfolio (9) and (11) in Proposition 3.1 is run on the five data sets J, K, L, M and N, respectively. Table 3 lists the final wealth $S_{2500}$ after 2500 trading days for selected values of the parameters $\eta, \beta$ and $\xi$ while fixing $c=0.1$. Table 3 also includes the final portfolio $\boldsymbol{b}_{2501}$ for each stock. The Table 4 gives the final wealth $S_{2500}$ for selected values of parameters $\eta, \xi, c_{1}$ and $c_{2}$ while fixing $d_{1}=1$ and $d_{2}=-1$. Table 4 also includes the final portfolio $\boldsymbol{b}_{2501}$ for each stock.

Table 2 gives the wealth achieved by running the Type 1 Helmbold universal portfolio on stock-price data sets J, K, L, M and N. The comparisons of the performance between Type 1 Helmbold universal portfolio, universal portfolio (9) and (11) is done.

By comparison, the result obtained from universal portfolio (9) and (11) are closed to the result from Type 1 Helmbold universal portfolio. The stock-price data sets J, K and M are good performing portfolios while stock-price data sets $\mathrm{L}$ and $\mathrm{N}$ are poor performing portfolios. However, in-term of wealth achieved, both universal portfolio (9) and (11) perform slightly better for stock-price data sets $\mathrm{J}, \mathrm{K}, \mathrm{L}$ and $\mathrm{M}$ as compare to Type 1 Helmbold universal portfolio. But Type 1 Helmbold universal portfolio perform better for stock-price data set N. However, the performances between Type I Helmbold universal portfolio, universal portfolio (9) and (11) do not show any significant difference for all stock-price data sets.

Table 1. List of Malaysian companies in data sets J, K, L, M and N.

\begin{tabular}{|c|c|}
\hline Data Set & Portfolio of Five Malaysian Companies \\
\hline $\mathbf{J}$ & $\begin{array}{l}\text { Public Bank, Nestle Malaysia, Telekom Malaysia, } \\
\text { Eco World Development Group, Gamuda }\end{array}$ \\
\hline K & $\begin{array}{l}\text { AMMB Holding, Air Asia, Encorp, } \\
\text { IJM Corp, Genting Plantations }\end{array}$ \\
\hline $\mathrm{L}$ & $\begin{array}{l}\text { Alliance Financial Group, DiGi.com, KSL Holdings, } \\
\text { IJM Corp, Kulim Malaysia }\end{array}$ \\
\hline $\mathrm{M}$ & $\begin{array}{c}\text { Hong Leong Bank, DiGi.com, Eco World Development Group, } \\
\text { Zecon, United Malacca }\end{array}$ \\
\hline $\mathrm{N}$ & $\begin{array}{l}\text { RHB Capital, Carlsberg Brewery Malaysia, KSL Holdings, } \\
\text { Crest Building Holdings, Kulim Malaysia }\end{array}$ \\
\hline
\end{tabular}


Table 2. The best wealth $S_{2500}$ obtained after 2500 trading days by running the Type 1 Helmbold portfolio (see [4]) over the data set J, K, L, M, N for selected value of $\gamma$ and the final portfolios after 2500 trading days $\boldsymbol{b}_{2501}$ are listed.

\begin{tabular}{|cccccccc|}
\hline Set & $\boldsymbol{\gamma}$ & $\boldsymbol{S}_{\mathbf{2 5 0 0}}$ & $\boldsymbol{b}_{\mathbf{1}}$ & $\boldsymbol{b}_{\mathbf{2}}$ & $\boldsymbol{b}_{\mathbf{3}}$ & $\boldsymbol{b}_{\mathbf{4}}$ & $\boldsymbol{b}_{\mathbf{5}}$ \\
\hline & 0.3 & 15.4957 & 0.126763 & 0.127786 & 0.114715 & 0.513119 & 0.117618 \\
$\mathrm{~J}$ & 0.4 & 16.1562 & 0.103203 & 0.104335 & 0.090455 & 0.608522 & 0.093485 \\
& 0.5 & 16.3094 & 0.082917 & 0.084071 & 0.070421 & 0.689242 & 0.073349 \\
& 0.6 & 15.9953 & 0.066127 & 0.067247 & 0.054443 & 0.755045 & 0.057138 \\
& 0.7 & 15.3086 & 0.052549 & 0.053598 & 0.041955 & 0.807542 & 0.044356 \\
\hline & -4.2 & 18.5711 & 0.068924 & 0.226932 & 0.646187 & 0.057511 & 0.000446 \\
$\mathrm{~K}$ & -4.1 & 18.6742 & 0.080392 & 0.263809 & 0.587355 & 0.067859 & 0.000585 \\
& -4.0 & 18.7101 & 0.092274 & 0.301252 & 0.526939 & 0.078781 & 0.000754 \\
& -3.9 & 18.6730 & 0.104260 & 0.338002 & 0.466762 & 0.090020 & 0.000956 \\
& -3.8 & 18.5588 & 0.116050 & 0.372854 & 0.408590 & 0.101310 & 0.001196 \\
\hline & -2.0 & 4.4220 & 0.261983 & 0.009207 & 0.504003 & 0.198672 & 0.026135 \\
$\mathrm{~L}$ & -1.9 & 4.4367 & 0.268802 & 0.011158 & 0.481837 & 0.208073 & 0.030130 \\
& -1.8 & 4.4449 & 0.274631 & 0.013474 & 0.460337 & 0.216948 & 0.034610 \\
& -1.7 & 4.4468 & 0.279396 & 0.016213 & 0.439609 & 0.225169 & 0.039613 \\
& -1.6 & 4.4429 & 0.283043 & 0.019440 & 0.419736 & 0.232612 & 0.045169 \\
\hline & 0.3 & 19.1103 & 0.114547 & 0.168995 & 0.489913 & 0.108616 & 0.117929 \\
& 0.4 & 19.7420 & 0.089628 & 0.150149 & 0.581495 & 0.085567 & 0.093161 \\
$\mathrm{M}$ & 0.5 & 19.9702 & 0.068846 & 0.130784 & 0.661432 & 0.066695 & 0.072243 \\
& 0.6 & 19.8071 & 0.052200 & 0.112292 & 0.728585 & 0.051626 & 0.055297 \\
& 0.7 & 19.3054 & 0.039230 & 0.095428 & 0.783593 & 0.039797 & 0.041952 \\
\hline & -2.3 & 4.9924 & 0.045748 & 0.199536 & 0.601143 & 0.134370 & 0.019203 \\
& -2.2 & 5.0073 & 0.050722 & 0.207542 & 0.575474 & 0.144158 & 0.022104 \\
& -2.1 & 5.0129 & 0.056009 & 0.214906 & 0.549948 & 0.153793 & 0.025344 \\
& -2.0 & 5.0087 & 0.061599 & 0.221550 & 0.524765 & 0.163140 & 0.028946 \\
& -1.9 & 4.9948 & 0.067479 & 0.227411 & 0.500107 & 0.172066 & 0.032937 \\
\hline
\end{tabular}

Table 3. The best wealth $S_{2500}$ obtained after 2500 trading days by running the universal portfolio generated by rational functions (9) over the data set $\mathrm{J}, \mathrm{K}, \mathrm{L}, \mathrm{M}, \mathrm{N}$ for selected value of $\eta, \beta, \xi, c=$ 0.1 and the final portfolios after 2500 trading days $\boldsymbol{b}_{2501}$ are listed.

\begin{tabular}{|cccccccccc|}
\hline Set & $\boldsymbol{\eta}$ & $\boldsymbol{\beta}$ & $\boldsymbol{\xi}$ & $\boldsymbol{S}_{\mathbf{2 5 0 0}}$ & $\boldsymbol{b}_{\mathbf{1}}$ & $\boldsymbol{b}_{\mathbf{2}}$ & $\boldsymbol{b}_{\mathbf{3}}$ & $\boldsymbol{b}_{\mathbf{4}}$ & $\boldsymbol{b}_{\mathbf{5}}$ \\
\hline & 1 & 1.1 & -2.0 & 17.2146 & 0.08107 & 0.08248 & 0.06965 & 0.69371 & 0.07309 \\
1 & 1.2 & -1.8 & 17.0865 & 0.08722 & 0.08859 & 0.07551 & 0.66976 & 0.07892 \\
& 1.1 & 1.2 & -1.8 & 16.9870 & 0.07815 & 0.07951 & 0.06662 & 0.70580 & 0.06992 \\
& 1.2 & 1.3 & -1.9 & 17.0492 & 0.07681 & 0.07819 & 0.06542 & 0.71081 & 0.06877 \\
$\mathrm{~J}$ & 0.9 & 1.3 & -2.0 & 17.0072 & 0.10518 & 0.10655 & 0.09366 & 0.59740 & 0.09721 \\
& 0.9 & 1.3 & -1.9 & 16.9640 & 0.10442 & 0.10578 & 0.09281 & 0.60067 & 0.09632 \\
& 1.1 & 1.4 & -2.0 & 17.2893 & 0.09165 & 0.09309 & 0.08016 & 0.65136 & 0.08374 \\
& 1.2 & 1.5 & -1.8 & 17.0051 & 0.08872 & 0.09007 & 0.07690 & 0.66400 & 0.08031 \\
& 1.4 & 1.5 & -2.0 & 16.9972 & 0.07455 & 0.07594 & 0.06325 & 0.71966 & 0.06660 \\
\hline
\end{tabular}




\begin{tabular}{|c|c|c|c|c|c|c|c|c|c|}
\hline \multirow{9}{*}{ K } & 1.2 & 1.3 & -2.0 & 19.6095 & 0.06344 & 0.05688 & 0.71233 & 0.06136 & 0.10599 \\
\hline & 1.1 & 1.2 & -1.9 & 19.4867 & 0.06473 & 0.05803 & 0.70703 & 0.06261 & 0.10760 \\
\hline & 1.1 & 1.1 & -1.9 & 19.4762 & 0.05750 & 0.05109 & 0.73634 & 0.05545 & 0.09962 \\
\hline & 1.5 & 1.7 & -1.9 & 19.0312 & 0.06552 & 0.05843 & 0.70328 & 0.06332 & 0.10945 \\
\hline & 1.2 & 1.8 & -2.0 & 19.0107 & 0.09106 & 0.08397 & 0.60272 & 0.08893 & 0.13332 \\
\hline & 1.6 & 2.0 & -2.0 & 18.9782 & 0.07350 & 0.06621 & 0.67127 & 0.07129 & 0.11773 \\
\hline & 1.5 & 1.8 & -1.9 & 18.9756 & 0.07042 & 0.06316 & 0.68358 & 0.06820 & 0.11464 \\
\hline & 1.5 & 2.1 & -2.0 & 18.9632 & 0.08322 & 0.07592 & 0.63304 & 0.08102 & 0.12680 \\
\hline & 1.4 & 1.9 & -1.9 & 18.9514 & 0.08107 & 0.07373 & 0.64147 & 0.07885 & 0.12488 \\
\hline \multirow{9}{*}{$\mathrm{L}$} & -2.0 & 1.8 & 0.2 & 6.5401 & 0.29548 & 0.12672 & 0.11370 & 0.29132 & 0.17278 \\
\hline & -2.0 & 1.7 & 0.2 & 6.5400 & 0.30137 & 0.12268 & 0.10878 & 0.29680 & 0.17037 \\
\hline & -2.0 & 2.9 & 0.4 & 6.5075 & 0.26050 & 0.15175 & 0.14401 & 0.25829 & 0.18545 \\
\hline & -2.0 & 2.9 & 1.0 & 6.4998 & 0.26957 & 0.14648 & 0.13415 & 0.26653 & 0.18327 \\
\hline & -2 & 1.2 & -0.1 & 6.4991 & 0.33894 & 0.09835 & 0.07858 & 0.33091 & 0.15322 \\
\hline & -2.0 & 1.2 & 0.2 & 6.4959 & 0.34511 & 0.09410 & 0.07449 & 0.33653 & 0.14977 \\
\hline & -1.7 & 1.1 & -0.4 & 6.4735 & 0.33217 & 0.10446 & 0.08140 & 0.32414 & 0.15783 \\
\hline & -1.8 & 1.1 & -0.3 & 6.4734 & 0.33903 & 0.09953 & 0.07695 & 0.33046 & 0.15403 \\
\hline & -1.6 & 1.1 & -0.6 & 6.4728 & 0.32350 & 0.11074 & 0.08722 & 0.31611 & 0.16243 \\
\hline \multirow{9}{*}{ M } & 1.1 & 1.3 & -2.0 & 21.0694 & 0.07179 & 0.13037 & 0.64779 & 0.07493 & 0.07512 \\
\hline & 1.0 & 1.2 & -1.9 & 20.9321 & 0.07395 & 0.13283 & 0.63966 & 0.07624 & 0.07732 \\
\hline & 1.1 & 1.4 & -1.9 & 20.9259 & 0.07759 & 0.13619 & 0.62557 & 0.07967 & 0.08098 \\
\hline & 1.2 & 1.2 & -2.0 & 20.8834 & 0.05664 & 0.11484 & 0.70818 & 0.06057 & 0.05977 \\
\hline & 1.3 & 1.9 & -2.0 & 20.8725 & 0.08749 & 0.14467 & 0.58770 & 0.08924 & 0.09090 \\
\hline & 1.2 & 1.8 & -2.0 & 20.8678 & 0.09061 & 0.14703 & 0.57589 & 0.09246 & 0.09401 \\
\hline & 1.3 & 2.7 & -1.9 & 19.9123 & 0.11553 & 0.16623 & 0.48505 & 0.11442 & 0.11877 \\
\hline & 1.7 & 2.8 & -2.0 & 19.9114 & 0.09769 & 0.15565 & 0.55081 & 0.09465 & 0.10120 \\
\hline & 1.6 & 2.6 & -1.9 & 19.8951 & 0.09692 & 0.15514 & 0.55374 & 0.09377 & 0.10043 \\
\hline \multirow{9}{*}{$\mathrm{N}$} & -2.0 & 1.1 & 1.9 & 4.6463 & 0.12884 & 0.24433 & 0.32404 & 0.21529 & 0.08750 \\
\hline & -1.9 & 1.1 & 1.8 & 4.6019 & 0.13276 & 0.24330 & 0.31569 & 0.21620 & 0.09205 \\
\hline & -2.0 & 1.3 & 1.2 & 4.4774 & 0.14128 & 0.23421 & 0.29101 & 0.22825 & 0.10525 \\
\hline & -1.9 & 1.3 & 1.8 & 4.4762 & 0.14342 & 0.23959 & 0.29428 & 0.21752 & 0.10519 \\
\hline & -1.9 & 1.3 & 1.9 & 4.4755 & 0.14381 & 0.24047 & 0.29472 & 0.21576 & 0.10524 \\
\hline & -2.0 & 1.5 & 1.9 & 4.4153 & 0.14840 & 0.23740 & 0.28484 & 0.21765 & 0.11171 \\
\hline & -2.0 & 1.5 & 1.8 & 4.4140 & 0.14816 & 0.23649 & 0.28419 & 0.21930 & 0.11186 \\
\hline & -2.0 & 1.5 & 1.7 & 4.4112 & 0.14805 & 0.23555 & 0.28334 & 0.22088 & 0.11218 \\
\hline & -2.0 & 1.5 & 1.6 & 4.4070 & 0.14807 & 0.23461 & 0.28231 & 0.22236 & 0.11265 \\
\hline
\end{tabular}

Table 4. The best wealth $S_{2500}$ obtained after 2500 trading days by running the universal portfolio generated by rational functions (11) over the data set J, K, L, M, N for selected value of $\eta, \xi, c_{1}, c_{2}$ and the final portfolios after 2500 trading days $\boldsymbol{b}_{2501}$ are listed.

\begin{tabular}{|ccccccccccc|}
\hline Set & $\boldsymbol{\eta}$ & $\boldsymbol{\xi}$ & $\boldsymbol{c}_{\mathbf{1}}$ & $\boldsymbol{c}_{\mathbf{2}}$ & $\boldsymbol{S}_{\mathbf{2 5 0 0}}$ & $\boldsymbol{b}_{\mathbf{1}}$ & $\boldsymbol{b}_{\mathbf{2}}$ & $\boldsymbol{b}_{\mathbf{3}}$ & $\boldsymbol{b}_{\mathbf{4}}$ & $\boldsymbol{b}_{\mathbf{5}}$ \\
\hline & 1.2 & 1.5 & -2.0 & 0.1 & 17.2924 & 0.08892 & 0.09036 & 0.07742 & 0.66227 & 0.08103 \\
& 1.1 & 1.4 & -1.9 & 0.1 & 17.1842 & 0.09122 & 0.09262 & 0.07960 & 0.65344 & 0.08312 \\
& 1.4 & 1.8 & -2.0 & 0.1 & 17.1534 & 0.08971 & 0.09112 & 0.07809 & 0.65945 & 0.08163 \\
& 1.4 & 1.7 & -2.0 & 0.1 & 17.1461 & 0.08497 & 0.08638 & 0.07340 & 0.67836 & 0.07689 \\
$\mathrm{~J}$ & 1.1 & 1.5 & -1.9 & 0.1 & 17.1378 & 0.09688 & 0.09828 & 0.08525 & 0.63078 & 0.08881
\end{tabular}




\begin{tabular}{|c|c|c|c|c|c|c|c|c|c|c|}
\hline & 1.3 & 1.9 & -2.0 & 0.1 & 17.1356 & 0.10075 & 0.10217 & 0.08926 & 0.61494 & 0.09288 \\
\hline & 1.2 & 1.4 & -1.9 & 0.1 & 17.1351 & 0.08297 & 0.08437 & 0.07142 & 0.68638 & 0.07486 \\
\hline & 1.2 & 1.6 & -1.9 & 0.1 & 17.1335 & 0.09403 & 0.09543 & 0.08237 & 0.64226 & 0.08591 \\
\hline & 0.9 & 1.1 & -1.9 & 0.1 & 17.1318 & 0.09080 & 0.09219 & 0.07910 & 0.65535 & 0.08256 \\
\hline \multirow{9}{*}{ K } & 1.2 & 1.3 & -2.0 & 0.1 & 19.6095 & 0.06344 & 0.05688 & 0.71233 & 0.06136 & 0.10599 \\
\hline & 1.3 & 1.3 & -2.0 & 0.1 & 19.5290 & 0.05584 & 0.04957 & 0.74313 & 0.05383 & 0.09764 \\
\hline & 1.4 & 1.5 & -2.0 & 0.1 & 19.4671 & 0.06079 & 0.05423 & 0.72284 & 0.05872 & 0.10342 \\
\hline & 1.2 & 1.5 & -2.0 & 0.1 & 19.4637 & 0.07556 & 0.06866 & 0.66376 & 0.07343 & 0.11859 \\
\hline & 1.4 & 1.7 & -2.0 & 0.1 & 19.3886 & 0.07136 & 0.06444 & 0.68030 & 0.06922 & 0.11468 \\
\hline & 1.3 & 1.4 & -1.9 & 0.1 & 19.3812 & 0.06178 & 0.05511 & 0.71877 & 0.05968 & 0.10466 \\
\hline & 1.0 & 1.1 & -1.8 & 0.1 & 19.3583 & 0.06620 & 0.05935 & 0.70097 & 0.06405 & 0.10943 \\
\hline & 1.0 & 1.2 & -1.9 & 0.1 & 19.3573 & 0.07418 & 0.06719 & 0.66916 & 0.07201 & 0.11746 \\
\hline & 1.1 & 1.2 & -1.8 & 0.1 & 19.3531 & 0.06435 & 0.05755 & 0.70843 & 0.06221 & 0.10746 \\
\hline \multirow{9}{*}{$\mathrm{L}$} & -2.0 & 1.8 & 0.2 & 0.1 & 6.5401 & 0.29548 & 0.12672 & 0.11370 & 0.29131 & 0.17279 \\
\hline & -2.0 & 1.9 & 0.3 & 0.1 & 6.5390 & 0.29207 & 0.12909 & 0.11655 & 0.28813 & 0.17416 \\
\hline & -2.0 & 1.9 & 0.2 & 0.1 & 6.5389 & 0.29020 & 0.13036 & 0.11817 & 0.28639 & 0.17488 \\
\hline & -2.0 & 2.0 & 0.5 & 0.1 & 6.5357 & 0.29105 & 0.12998 & 0.11720 & 0.28710 & 0.17466 \\
\hline & -2.0 & 1.6 & -0.2 & 0.1 & 6.5356 & 0.30039 & 0.12360 & 0.10921 & 0.29584 & 0.17096 \\
\hline & -2.0 & 1.4 & 0.1 & 0.1 & 6.5274 & 0.32194 & 0.10901 & 0.09203 & 0.31571 & 0.16131 \\
\hline & -2.0 & 1.5 & -0.5 & 0.1 & 6.5273 & 0.30249 & 0.12263 & 0.10685 & 0.29765 & 0.17038 \\
\hline & -1.9 & 1.4 & -0.1 & 0.1 & 6.5215 & 0.31457 & 0.11451 & 0.09704 & 0.30876 & 0.16512 \\
\hline & -2.0 & 2.6 & 0.4 & 0.1 & 6.5185 & 0.26780 & 0.14639 & 0.13751 & 0.26523 & 0.18307 \\
\hline \multirow{9}{*}{ M } & 1.1 & 1.3 & -2.0 & 0.1 & 21.0694 & 0.07179 & 0.13037 & 0.64779 & 0.07492 & 0.07513 \\
\hline & 1.0 & 1.1 & -1.9 & 0.1 & 20.9148 & 0.06659 & 0.12567 & 0.66853 & 0.06933 & 0.06988 \\
\hline & 1.1 & 1.2 & -1.9 & 0.1 & 20.9099 & 0.06456 & 0.12359 & 0.67655 & 0.06747 & 0.06783 \\
\hline & 1.2 & 1.2 & -2.0 & 0.1 & 20.8834 & 0.05664 & 0.11484 & 0.70818 & 0.06057 & 0.05977 \\
\hline & 1.3 & 1.9 & -2.0 & 0.1 & 20.8725 & 0.08749 & 0.14467 & 0.58770 & 0.08924 & 0.09090 \\
\hline & 1.4 & 2.0 & -2.0 & 0.1 & 20.8071 & 0.08497 & 0.14292 & 0.59741 & 0.08630 & 0.08840 \\
\hline & 1.0 & 1.1 & -1.8 & 0.1 & 20.8060 & 0.06610 & 0.12549 & 0.67073 & 0.06829 & 0.06939 \\
\hline & 1.1 & 1.3 & -1.8 & 0.1 & 20.8048 & 0.07101 & 0.13046 & 0.65140 & 0.07276 & 0.07437 \\
\hline & 1.0 & 1.4 & -1.9 & 0.1 & 20.8038 & 0.08702 & 0.14446 & 0.58966 & 0.08844 & 0.09042 \\
\hline \multirow{9}{*}{$\mathrm{N}$} & -2.0 & 1.1 & 1.9 & 0.1 & 4.6463 & 0.12884 & 0.24433 & 0.32404 & 0.21529 & 0.08750 \\
\hline & -2.0 & 1.2 & 1.7 & 0.1 & 4.5730 & 0.13462 & 0.24055 & 0.30887 & 0.22084 & 0.09512 \\
\hline & -1.9 & 1.2 & 1.9 & 0.1 & 4.5339 & 0.13892 & 0.24238 & 0.30445 & 0.21519 & 0.09906 \\
\hline & -1.9 & 1.3 & 1.9 & 0.1 & 4.4755 & 0.14381 & 0.24047 & 0.29472 & 0.21576 & 0.10524 \\
\hline & -2.0 & 1.4 & 1.9 & 0.1 & 4.4626 & 0.14454 & 0.23911 & 0.29212 & 0.21762 & 0.10661 \\
\hline & -2.0 & 1.4 & 1.8 & 0.1 & 4.4614 & 0.14430 & 0.23815 & 0.29137 & 0.21941 & 0.10677 \\
\hline & -2.0 & 1.4 & 1.3 & 0.1 & 4.4337 & 0.14508 & 0.23346 & 0.28502 & 0.22659 & 0.10985 \\
\hline & -1.9 & 1.4 & 1.8 & 0.1 & 4.4249 & 0.14763 & 0.23778 & 0.28629 & 0.21760 & 0.11070 \\
\hline & -2.0 & 1.4 & 1.2 & 0.1 & 4.4248 & 0.14557 & 0.23264 & 0.28346 & 0.22751 & 0.11082 \\
\hline
\end{tabular}




\section{References}

1. T.M. Cover, E. Ordentlich, IEEE Trans. Inform. Theory 42(2), 348 - 363 (1996)

2. C.P. Tan, Performance bounds for the distribution-generated universal portfolios, in Proceedings 59th ISI World Statistics Congress, Hong Kong, 5327 - 5332 (2013)

3. D.P. Helmbold, R.E. Shapire, Y. Singer and M.K. Warmuth, Math. Financ. 8(4), 325 347 (1998)

4. C.P. Tan, K.S. Kuang, IOSR JM 14, 19 - 25 (2018)

5. C.P. Tan, K.S. Kuang, IOSR JM 15, 67 - 72 (2019)

6. L. Pardo, Statistical inference based on divergence measures (FL: CRC Press, Boca Rotan, 2006) 